\title{
EXPLODING INTERSTELLAR GRAINS AND COMPLEX MOLECULES
}

\author{
J. MAYO GREENBERG \\ State University of New York at Albany \\ and \\ Dudley Observatory \\ and
}

ANDREW J. YENCHA

State University of New York at Albany

\begin{abstract}
The triggering of an explosive chemical reaction in a grain which consists of free radicals frozen in a matrix of photolyzed dirty ice material is seen as a possible source of complex interstellar organic molecules.
\end{abstract}

\section{Introduction}

The interstellar dust appears to play a critical role in the formation of the interstellar molecules. As is shown elsewhere in this volume the rate of formation of complex molecules directly out of the gas fails by orders of magnitude to compete with the rate of destruction in maintaining the observed abundances. Exactly what the mechanism of molecule production via dust may be is not clearly defined. But it seems clear that the chemical and physical character of the dust are critical in distinguishing between the relative importance of the various conceivable processes.

The distribution of the molecules under consideration is sufficiently far removed from stars that they can only have reached the regions in times much larger than their typical decay times as determined by the ultraviolet (or other disruptive) radiation.

A molecule with a radiative life limited to $100 \mathrm{yr}$ in a radiation field like that suggested by Habing would travel, at a speed of $10 \mathrm{~km} \mathrm{~s}^{-1}$, a distance of merely $10^{-3} \mathrm{pc}$ before being destroyed. The existence of reasonably complex molecules well beyond this distance from the center of dark clouds seems to preclude the likelihood of their formation in the atmosphere of protostars.

Molecules may be formed on or in grain surfaces. The surface phenomenon has been a subject of both theoretical and experimental studies (Breuer, 1973). The possibility of molecules being an intrinsic part of the grain and appearing in space as the result of some grain disruption process or event is the focal point of this investigation. Both laboratory as well as theoretical investigation bearing on this process are considered.

\section{Radiation Effects on Dust}

For our basic dust model we consider those interstellar grains which have formed by 
accretion of $\mathrm{O}, \mathrm{C}$. and $\mathrm{N}$ atoms in combination with hydrogen to form a dirty ice mantle on some core - generally a silicate.

The significance of radiation effects on the dust is deternined by the time it takes for all the molecules in a dust grain to have absorbed a photon compared with characteristic dust lifetimes which are of the order of $10^{7} \mathrm{yr}$. The number of photons impinging per second on a dust grain of radius $a$ is

$$
\frac{\mathrm{d} N_{h v}}{\mathrm{~d} t}=\frac{\pi a^{2}}{h} \int_{912}^{\lambda_{t}} u_{\lambda} \mathrm{d}_{\lambda}
$$

which, for the distribution we are using $u \lambda=40 \times 10^{-18} \mathrm{erg} \mathrm{cm}^{-3}, 912>\lambda>2000 \AA$, (approximated from Habing, 1968), gives

$$
\frac{\mathrm{d} N_{h v}}{\mathrm{~d} t}=30 \pi a^{2}\left(\lambda_{t}^{2}-912^{2}\right),
$$

where $\lambda_{t}$ is in ångströms.

Thus for $\lambda_{t} \leq 2000 \AA$ the number of photons impinging on a $0.1 \mu$ size grain is

$$
\frac{\mathrm{d} N_{h v}}{\mathrm{~d} t} \leq 33 \times 10^{3} \mathrm{~s}^{-1} .
$$

If we assume that the absorption efficiency of the grains is of the order of one, the total time for every molecule to have been subjected to a bondbreaking photon is $\left(\mathrm{d} N_{h \mathrm{v}} / \mathrm{d} t / n_{\text {mol }}\right)^{-1}$ where $n_{\text {mol }}$ is the number of molecules in the grain. For an average molecular size $\simeq 2 \AA$, we find the saturation time to be of the order of $130 \mathrm{y}$. This is indeed very short compared with $10^{7} \mathrm{yr}$.

As a consequence of the ultraviolet irradiation of the interstellar grains a number of phenomena take place (Bass and Broida, 1960), among which are the formation and trapping of free radicals, and the formation of complex organic molecules. This latter problem has been investigated by many workers largely inspired by the initial work on the prebiogenic synthesis of organic molecules in the Earth's atmosphere (see R. M. Lemon, 1970 for a bibliography). The very low temperature solid state conditions prevailing in interstellar grains have not been considered in this connection for obvious reasons. However, low temperature solids have sufficiently low diffusion rates that they are most ideally suited for the trapping of free radicals.

The variety of possible processes to be considered are: (1) Creation of free radicals by either bond-breaking or ionization (we will limit ourselves here to bond-breaking), (2) immediate recombination of the original molecule, (3) migration of the free radical, (4) recombination of different radicals, (5) combination of radicals with molecules. The latter process is like polymerization.

Schematically the system is represented by (as modified from Morawetz, 1960): radical production $m \rightarrow r \quad$ rate: $p$, growth of large unsaturated molecules: $M+r \rightarrow r$ rate: $k_{g}[M][r]$, termination to saturated molecules: $r+r \rightarrow S$ rate: $k_{t}[r]^{2}$, 
where $[M]$ and $[r]$ are the number of reacting molecules and free radicals.

In steady state

$$
\begin{aligned}
& \frac{\mathrm{d}[r]}{\mathrm{d} t}=p-k_{t}[r]^{2}=0 \\
& {[r]=\left(p / k_{t}\right)^{\frac{1}{2}} .}
\end{aligned}
$$

The rate of consumption of 'reactive' molecules is

$$
\frac{\mathrm{d}[M]}{\mathrm{d} t}=\left(k_{g} / k_{t}^{\frac{1}{2}}\right)[M] p^{\frac{1}{2}}
$$

and the average number $\bar{s}$, of 'reactive' molecules in the large molecules, $S$, is given by

$$
\bar{s}=-\frac{\mathrm{d}[M] / \mathrm{d} t}{\mathrm{~d}[S] / \mathrm{d} t}=f k_{g}\left(k_{t} p\right)^{-\frac{1}{2}}[M],
$$

where $f$ is a fraction varying between $\frac{1}{2}$ and 1 .

We have already shown that $p \approx 2.5 \times 10^{-10}$. In order to achieve a reasonable concentration of free radicals we see that the recombination rate must also be of this order. This rate will depend on the molecular composition already achieved because it will be significantly less as the molecule and radical sizes increase to produce reduced diffusion rates. In some cases ESR measurements have indicated that, subsequent to irradiation, free radical concentration remained unchanged for a month (Bijl and RoseInnes, 1955) implying quite small values of $k_{t}$.

The two extreme possibilities for a grain are that either it maintains a high concentration of free radicals or it forms predominantly saturated complex molecules. It should be noted that from a purely theoretical point of view the absolute maximum of free radical concentration cannot be greater than about $10 \%(\mathrm{Jackson}$ and Montroll, 1958). Experimentally the maximum values achieved are less than $1 \%$. However, there does not appear to be sufficient data or theory at the present to exclude the possibility of substantial free radical concentration in the grains sufficient to give rise to exotic optical properties (Greenberg et al., 1971). In any case the formation of large organic molecules is certain to take place during irradiation. It is interesting to note that if a grain containing $1 \%$ free radical concentration is triggered to ignition of a chain chemical reaction, the total energy released is sufficient to evaporate the grain.

With this process of grain disruption by evaporation we may anticipate the presence of a wide range of complex organic molecules, many appearing originally with extremely high molecular weights. Subsequent degradation of the initially very large molecules would occur as a result of photon disruption. On this basis the molecules observed would be the resulting products of large molecule breakup rather than the growth from smaller molecules either in space or on grain surfaces. The equilibrium density of molecules is then given by equating the creation rate for grain disruption with the destruction rate by radiation. We have then equality between 


$$
\left(\frac{\mathrm{d} N_{\text {mol }}}{\mathrm{d} t}\right)_{\text {creation }}=n_{\text {mol }} N_{d} / \tau_{d}
$$

and

$$
\left(\frac{\mathrm{d} N_{\text {mol }}}{\mathrm{d} t}\right)_{\text {destruction }}=N_{\text {mol }} / \tau_{\text {mol }},
$$

where $n_{\text {mol }}=$ number of molecules per dust grain, $N_{d}=$ number of dust grains per $\mathrm{cm}^{3}, \tau_{d}=$ disruption lifetime for dust and $\tau_{\mathrm{mol}}=$ radiative lifetime of a typical molecule, and $N_{\text {mol }}=$ number of molecules per $\mathrm{cm}^{3}$ in space.

Using a grain size of $0.1 \mu$ and a ratio of dust to hydrogen density of $10^{-2}$ we get

$$
\tau_{d}=4 \times 10^{-3} \tau_{\mathrm{mol}} N_{\mathrm{H}} / N_{\mathrm{mol}} .
$$

In dense clouds, where molecules are found, $\tau_{\text {mol }}$ may be of the order of $10^{4} \mathrm{yr}$ or greater and $N_{\mathrm{H}} / N_{\text {mol }} \approx 10^{-8}$. For these values $\tau_{d}$ is of the order of $4 \times 10^{9} \mathrm{yr}$ which is extremely large compared with the formation and growth lifetimes of dust, and would not deplete the dust density significantly.

Photochemical experiments have been made on simulated interstellar materials. In the initial stages of our studies we have irradiated solid mixtures of water, methane (or ethane), and ammonia with non-ionizing radiation between 1400 and $2000 \AA$ wavelength and have looked at the product distribution using a mass spectrometer. The experiments are carried out by preparing various mixtures of gaseous water, methane or ethane and ammonia in 'cosmic abundance' proportions of oxygen, carbon, and nitrogen, and then depositing the mixture on a cold finger in a cryostat. After depositing approximately $20 \mathrm{mg}$ of the mixture onto the cold finger, which results in a 'dirty ice' pattern of about $25 \mathrm{~mm}$ in diameter, the radiation source (Cary hydrogen arc lamp fitted with a sapphire window) is turned on. The pressure in the system is maintained below $10^{-6}$ torr throughout the irradiation process. We have performed experiments at 28, 42, and $77 \mathrm{~K}$ with irradiation times varying between 4 and $6 \mathrm{~h}$. The experiments at $77 \mathrm{~K}$ required the use of ethane instead of methane since methane has an appreciable vapor pressure at this temperature. After irradiation is complete the cold finger is allowed to warm up to room temperature and the sample cryogenically pumped into an evacuated tube and subsequently injected into a mass spectrometer (AEI ms $902 \mathrm{Cl}$ ) for analysis. The mass spectra of the samples clearly indicate the presence of high molecular weight (mass $>106$ and possibly some with mass $>200$ ) organic material. In order to insure against organic contamination we have employed a mercury diffusion pump with liquid nitrogen cold trapping between it and the mechanical pump and the system itself. Even so, we routinely run blanks before each experiment to check for possible contamination. In general, two blanks are taken. One consists of depositing a sample onto the cold finger and then removing it without any radiation. The second blank obtained by irradiation of the cold finger without any sample and subsequent collection of any material. Both of these blank samples are run on the mass spectrometer immediately prior to the analysis of our irradiated sample and the results compared with the mass spectrum of the irradiated sample. 
The results obtained thus far indicate the presence of numerous organic materials, the total amount of products being of the order of $10 \mu \mathrm{g}$ (implying a photon efficiency of the order of $0.1 \%$.) We have not been able to make positive identification of these species. The degree of complexity of the final composition of the material does not appear to depend sensitively on the temperature and the implication is that complex products will be produced at temperatures of $10 \mathrm{~K}$ or less with efficiencies which make the process astronomically important.

At present we are conducting our experiments using nitrogen-15 ammonia, deuterated methane, and deuterated water in further attempts to clarify our results. In addition, we have interfaced a gas chromatograph (Hewlett Packard 5750) with the mass spectrometer to enable us to separate our products prior to being mass analyzed. This should prove to be very helpful in our attempts to identify the irradiation products.

\section{References}

Bass, A. M. and Broida, H. P., (eds.): 1960, Formation and Trapping of Free Radicals, Academic Press, New York.

Bijl, D. and Rose-Innes, A. C.: 1955, Nature 175, 82.

Breuer, H. D.: 1973, this volume, p. 399.

Greenberg, J. M., Yencha, A. J., Corbett, J. W., and Frisch, H. L.: 1972, Mem. Soc. Roy. Sci. Liège, 6ème Sér. 3.

Jackson, J. L. and Montroll, E. W.: 1958, J. Chem. Phys. 28, 1101. 